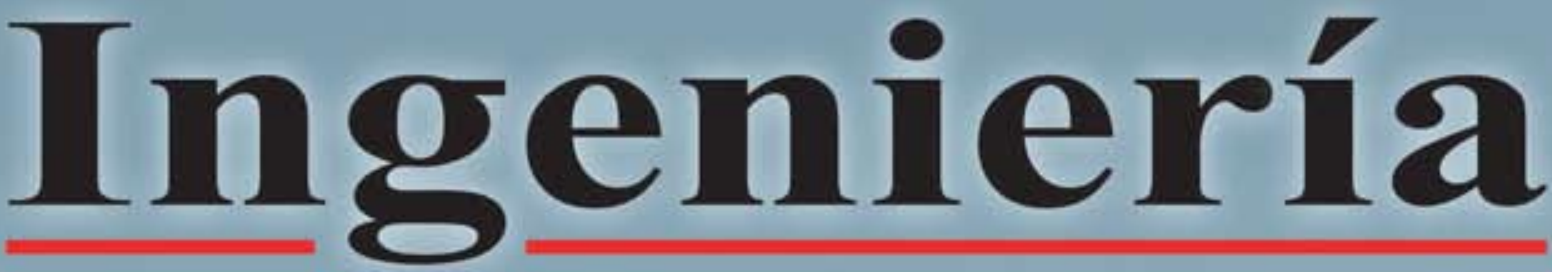

ENERO/DICIEMBRE 2004 - VOLUMEN 14 - N¹ y 2

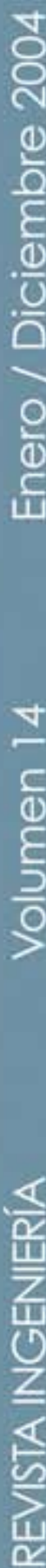
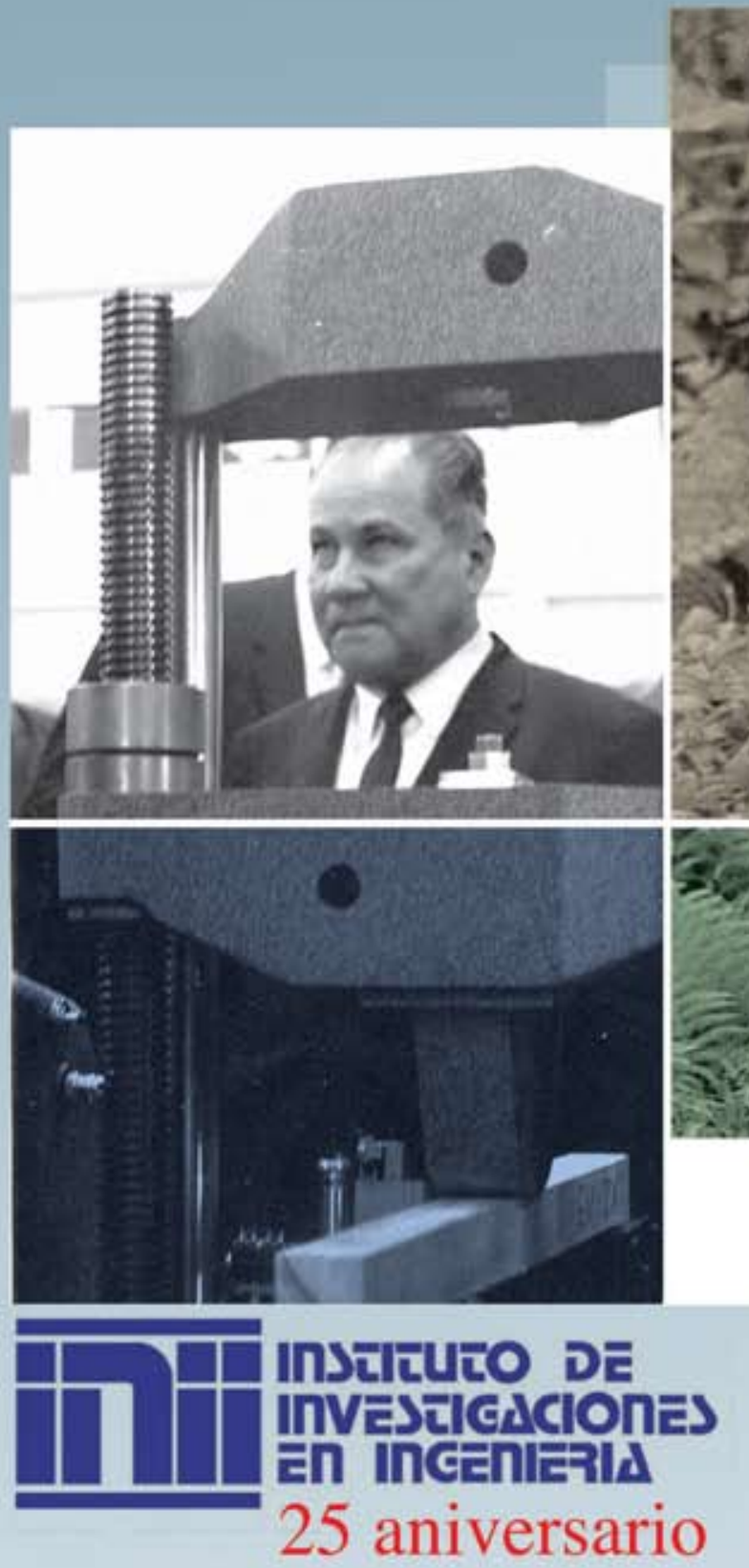

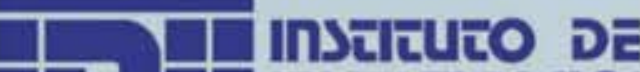
T W Investcscines हn Incenlei|d 25 aniversario

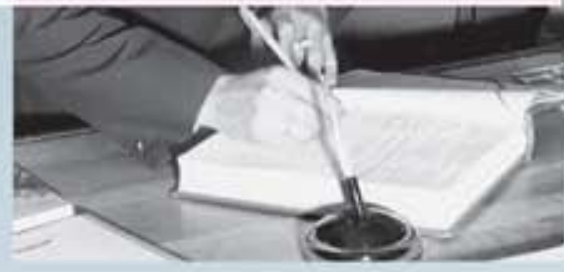




\title{
CÓDIGO SÍSMICO DE COSTA RICA VERSIÓN 2002. FACTORES DE REDUCCIÓN DE RESISTENCIA POR DUCTILIDAD
}

\begin{abstract}
Resumen
Este artículo constituye la primera parte de una serie de tres investigaciones cuyo objetivo es validar las formas espectrales que se utilizan para el diseño sismorresistente de las estructuras civiles definidas en la nueva versión del Código Sísmico de Costa Rica (CSCR-2002). La comparación de los diversos criterios existentes para obtener los factores de reducción de resistencia por ductilidad de desplazamiento, es realizada con el criterio de Riddell y Newmark (1979), el cual es el utilizado en los espectros de diseño inelásticos del CSCR-2002. El criterio de desamplificación del espectro de diseño elástico propuesto por Miranda (1993) presenta una mejor concordancia con el comportamiento esperado para los factores de reducción de resistencia. Las diferencias existentes entre ambos criterios se presentan en todas las ductilidades y en todos los rangos de períodos al realizar la comparación de los valores espectrales.
\end{abstract}

Palabras clave: ductilidad, espectros de diseño elástico, espectro de diseño inelástico, factores de reducción de resistencia, diseño sísmico, valores espectrales, código de diseño sísmico.

\begin{abstract}
This is the first part of a series of three investigations whose objective is to validate the spectral shapes that are the basis for the seismic-resistant design of the buildings defined into the new version of the Seismic Code of Costa Rica (CSCR-2002). The comparison between the existent criteria to obtain the strength reduction factors by displacement ductility with respect to the Riddel and Newmark's criterion (1979), which is the basis for the inelastics design spectra of the CSCR-2002, was made. The desamplification concept referred to the elastic design spectra proposed by Miranda (1993) presents a better fitting with the strength reduction factors'expected behavior. The differences between both criteria are present in all the ductilities and in all the period ranges, in the moment of the comparison between the spectral values.
\end{abstract}

Keywords: ductility, elastic design spectra, inelastic design spectra, strength reduction factors, seismic design, spectral values, seismic design code.

\section{Recibido 2004-VII-08 / Aceptado 2004-X-04}

\section{INTRODUCCIÓN}

Los factores de reducción de la resistencia permiten la evaluación de las demandas de resistencia inelásticas a partir de las demandas de resistencias elásticas. Reducciones en las fuerzas laterales producidas por la capacidad de disipación de la energía histerética de una estructura, son expresadas por los factores de reducción de resistencia o por sus recíprocos (factores de desamplificación). Lograr proveer a la estructura de una capacidad de resistencia lateral mínima, que resulte en un control adecuado de las deformaciones inelásticas durante eventos sísmicos fuertes, relacionadas con el concepto de daño, requiere una buena estimación de los factores de reducción de resistencia.

\section{DUCTILIDAD DE DESPLAZAMIENTO}

La razón de ductilidad de desplazamiento se define como el nivel de deformación inelástica experimentado por un sistema estructural sometido a un movimiento fuerte del terreno. La siguiente ecuación la expresa en términos de la razón del desplazamiento relativo máximo con respecto a su desplazamiento de fluencia 
$\mu=\frac{\max |u(t)|}{u_{y}}$

donde $\mu$ es la ductilidad de desplazamiento, $u(t)$ es la historia en el tiempo del desplazamiento del sistema $u_{y}$ es el desplazamiento de fluencia del sistema.

El diseño sismorresistente se considera adecuado cuando, por medio de un detalle de los elementos que conforman el sistema estructural, se logran capacidades de ductilidad mayores que las demandadas por el evento sísmico máximo probable que actúa sobre éste. Esto implica que una estructura con una resistencia de fluencia dada $F_{y}$ la cual disipa energía por medio de deformación inelástica, debe tener una capacidad de deformación límite que exceda la demanda sísmica, en el caso de sismos fuertes.

Partiendo de la ecuación de movimiento $\left(m \ddot{u}+c \dot{u}+k u=-m \ddot{u}{ }_{g}(t)\right)$ que plantea el equilibrio dinámico de un sistema de un grado de libertad, e incorporando la razón de ductilidad de desplazamiento, y la relación de la fuerza elástica con respecto a la fuerza en el punto de fluencia del sistema $(\rho(t))$, se logra construir una expresión que explícitamente define el movimiento en términos de la razón de ductilidad, lo que, para efectos posteriores, permitirá una comparación con los espectros de diseño del CSCR-2002.

$\ddot{\mu}(t)+2 w \xi \dot{\mu}(t)+w^{2} \rho(t)=-\left(\frac{w^{2}}{\eta}\right) \frac{g}{\ddot{u}_{g, \max }}$ donde $w$ es la frecuencia natural de oscilación, $\xi$ es la razón de amortiguamiento.

\section{ESPECTRO DE DISEÑO DEPENDIENTE DEL SITIO}

Mohraz (1973) realizó un estudio estadístico detallado de las razones de los parámetros de movimiento del terreno V/A y $\mathrm{AD} / \mathrm{V}^{2}$, determinando que los valores para la razón V/A para roca son sustancialmente menores que los valores para suelo aluvional. Los valores obtenidos para la razón $\mathrm{AD} / \mathrm{V}^{2}$ indican que las relaciones para aluvión son más pequeñas que aquellas para roca. Al representar esta razón una medida del ancho del espectro, se infiere que los espectros para roca son más planos que aquellos para aluvión.

El Cuadro 1 presenta diferencias en los valores de las relaciones espectrales producidas por el efecto del sitio de cimentación.

\section{FACTORES DE MODIFICACIÓN DE LA RESPUESTA ELÁSTICA}

El factor de reducción de resistencia o factor de modificación de la respuesta $\mathrm{R}$ contempla cuatro subfactores: relaciones con la ductilidad (desplazamiento inelástico), sobrerresistencia (resistencia de la estructura real mayor a la utilizada en el diseño), amortiguamiento (mecanismos de disipación de movimiento) y redundancia (grados de indeterminación estática

Cuadro 1. Valores de relaciones espectrales para componentes horizontales.

Condición del sitio
Roca (Mohraz)
Estrato menor a 10m de aluvión sobre roca (Mohraz)
Estrato de 10m-65m de aluvión sobre roca (Mohraz)
Aluvión (Mohraz)
Roca (Hall)
Aluvión (Hall)

Valor promedio
V/A
27
37
33
51
28
48

Valor promedio
$\mathrm{AD} / \mathrm{V}^{2}$
6,9
5,2
5,6
4,3
6
6

Fuente: (Gupta, 1992) 
inherentes a la estructura). Para efectos de comparación sólo va a ser considerando el factor de reducción de resistencia por ductilidad de desplazamiento

$R_{\mu}=\frac{F_{y}(\mu=1)}{F_{y}\left(\mu=\mu_{i}\right)}$

donde $F_{y}$ es el valor de la fuerza en la fluencia del sistema.

\section{ESPECTRO DE DISEÑO INELÁSTICO}

El espectro de diseño inelástico se obtiene a partir de la reducción del espectro de diseño elástico por medio del uso de los factores de modificación o factores de reducción dependientes de la ductilidad.

Las estructuras sujetas a severos movimientos del terreno experimentan deformaciones más allá del rango elástico. Estas deformaciones inelásticas dependen de las características de carga-deformación y a menudo resultan en deterioro de la rigidez. Debido a la naturaleza cíclica del movimiento del terreno, las estructuras experimentan etapas sucesivas de carga y descarga, y la relación de fuerza-desplazamiento o resistencia-deformación siguen una secuencia de ciclos conocido como ciclo histerético. Los ciclos son una medida de la capacidad de la estructura para disipar energía. La forma y orientación de los ciclos histeréticos dependen primeramente de la rigidez de la estructura y del desplazamiento de fluencia. Factores tales como el material estructural, el sistema estructural y la configuración de las conexiones influyen en el comportamiento histerético de la estructura.

Newmark (1973) propone un método para la construcción del espectro inelástico a partir del conocimiento de las regiones espectrales en las cuales observa lo siguiente: para períodos largos el sistema elástico y el sistema inelástico tienen el mismo desplazamiento total; para períodos intermedios los sistemas elástico e inelástico absorben la misma energía total y para períodos cortos, ambos sistemas poseen la misma fuerza. Estas consideraciones de igual desplazamiento, igual energía e igual aceleración, le permiten a Newmark recomendar dividir las ordenadas del espectro elástico de diseño por dos coeficientes que dependen de la razón de ductilidad de desplazamiento, Figura 1.

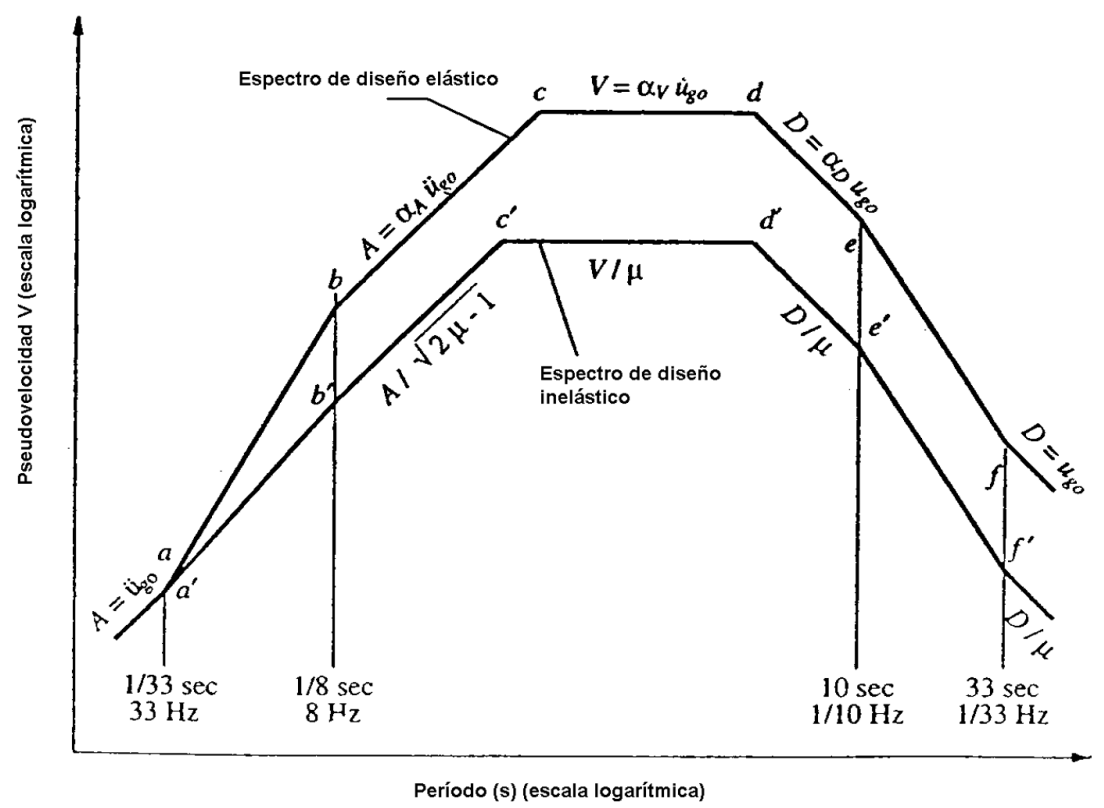

Figura 1. Factores de construcción del espectro inelástico de diseño según Newmark (Chopra, 1981). 


\section{CRITERIOS DE OBTENCIÓN DE FACTORES DE REDUCCIÓN DE RESISTENCIA POR DUCTILIDAD}

Es importante notar que por sí sola la ductilidad no se constituye en el factor de reducción, es el paso inicial para obtener el factor que reduce o desamplifica la respuesta elástica del sistema de un grado de libertad, para tomar en cuenta la incursión inelástica del sistema estructural. A continuación se detallan, en estricto orden cronológico, los criterios que se han definido para la obtención de los factores de reducción de resistencia

\subsection{Newmark y Veletsos (1960)}

Establecen la primera recomendación para los factores de reducción. Ellos indicaban la importancia de relacionar la deformación máxima de un sistema elastoplástico con aquel de un sistema elástico bajo la premisa de tener un valor de rigidez elástico igual al valor de la rigidez inicial del sistema inelástico. Lograron determinar que para el rango de bajas frecuencias con valores menores a $0,38 \mathrm{~Hz}$ (zona de períodos mayores a 16,5 s) la máxima deformación del sistema inelástico y su sistema asociado elástico eran muy similares. Este descubrimiento sentó las bases de la regla de igual deformación. De forma similar, notaron que para la zona de frecuencias altas o moderadamente altas (períodos bajos) los desplazamientos inelásticos eran significativamente superiores a sus contrapartes elásticas.

\subsection{Newmark y Hall (1973)}

Utilizan como base teórica los resultados del trabajo de Newmark y Veletsos. Reducen el espectro elástico por factores diferentes para cada región espectral, de acuerdo con las siguientes consideraciones:

1. Para las regiones espectrales de períodos altos o moderadamente altos, los sistemas elásticos e inelásticos tienen aproximadamente el mismo desplazamiento máximo.

2. En la región de períodos muy bajos los sistemas elásticos e inelásticos tienen aproximadamente la misma fuerza.

3. En la región de períodos moderadamente bajos el principio de conservación de la energía puede ser utilizado para explicar la similitud entre el diagrama de carga monotónica versus deformación del sistema elástico llevado al nivel de deformación máxima con el mismo diagrama, pero para un sistema elastoplástico.

Se normaliza un conjunto de registros de aceleración con respecto a sus valores máximos calculando el espectro de respuesta para cada registro normalizado, asumiendo varios coeficientes de amortiguamiento, para finalmente evaluar y suavizar el valor numérico de los casos del valor medio y del valor medio más una desviación estándar (50 percentil y 84,1 percentil de una distribución normal respectivamente) para cada amortiguamiento. Los espectros inelásticos de diseño se obtienen por medio de la desamplificación de las ordenadas elásticas por medio de los factores de reducción de resistencia dependiente de la ductilidad.

La definición de estos valores se presenta a continuación:

$$
0 \leq T \leq \frac{T_{1}}{10} \quad R_{\mu}=1
$$

$\frac{T_{1}}{10} \leq T \leq \frac{T_{1}}{4} \quad R_{\mu}=\sqrt{2 \mu-1}\left[\frac{T_{1}}{4 T}\right]^{2,513 \log \left[\frac{1}{\sqrt{2 \mu-1}}\right]}$

$\frac{T_{1}}{4} \leq T \leq T_{1}^{\prime} \quad R_{\mu}=\sqrt{2 \mu-1}$

$T_{1}^{\prime} \leq T \leq T_{1} \quad R_{\mu}=\frac{T \mu}{T_{1}}$

$T_{1} \leq T \leq T_{2} \quad R_{\mu}=\mu$

$T_{2} \leq T \leq 10 s \quad R_{\mu}=\mu$ 
Las tres regiones espectrales se encuentran limitadas por los valores de los períodos esquinas definidos en las siguientes ecuaciones:

$$
\begin{aligned}
& T_{1}=2 \pi \frac{\alpha_{v}}{\alpha_{a}} \frac{V}{A} \\
& T_{1}^{\prime}=T_{1} \frac{\mu}{\sqrt{2 \mu-1}} \\
& T_{2}=2 \pi \frac{\alpha_{d}}{\alpha_{v}} \frac{D}{V}
\end{aligned}
$$

A, V, D son los valores máximos de aceleración, velocidad y desplazamiento del terreno; $\alpha_{a}, \alpha_{v}, \alpha_{d}$ son los factores de amplificación que, aplicados a los parámetros del movimiento máximo del terreno, dan las ordenadas del espectro de diseño elástico, en las regiones espectrales de aceleración, velocidad y desplazamiento respectivamente. Para un amortiguamiento del $5 \%$, se tiene los valores de $\alpha_{a}=2,6, \alpha_{v}=1,9$ y $\alpha_{d}=1,4$.

\subsection{Riddell y Newmark (1979)}

Determinan que el uso del sistema elastoplástico proporciona resultados conservadores para el espectro inelástico. Dentro de las formulaciones para el cálculo de los factores de reducción se encuentra incorporada la razón de amortiguamiento según fórmula 6 .

Los valores de los períodos esquina son:

$$
\begin{aligned}
& T_{1}=2 \pi \frac{\alpha_{v}}{\alpha_{a}} \frac{V}{A} \\
& T_{1}^{\prime}=T_{1} \frac{\left(p_{a} \mu-q_{a}\right)^{a_{a}}}{\left(p_{v} \mu-q_{v}\right)^{v_{v}}} \\
& T_{2}=2 \pi \frac{\alpha_{d}}{\alpha_{v}} \frac{D}{V} \\
& T_{2}^{\prime}=T_{2} p_{d} \mu^{-r_{d}}\left(p_{v} \mu-q_{v}\right)^{r_{v}}
\end{aligned}
$$

Para un amortiguamiento del $5 \%$, se tiene los valores de $\alpha_{a}=2,77, \alpha_{v}=2,15$ y $\alpha_{d}=2,10$.

$$
\begin{array}{lll}
0 \leq T \leq 0,0303 & R_{\mu}=1 \\
0,0303 \leq T \leq 0,125 & R_{\mu}=\left(p_{a} \mu-q_{a}\right)^{a}\left[\frac{1}{8 T}\right]^{1,625 \log \left[\left(p_{a} \mu-q_{a}\right)^{-r_{a}}\right]} \\
0,125 \leq T \leq T_{1}^{\prime} & R_{\mu}=\left(p_{a} \mu-q_{a}\right)^{x^{a}} & \\
T_{1}^{\prime} \leq T \leq T_{1} & R_{\mu}=\frac{T}{T_{1}}\left(p_{v} \mu-q_{v}\right)^{r_{v}} & \\
T_{1} \leq T \leq T_{2}^{\prime} & R_{\mu}=\left(p_{v} \mu-q_{v}\right)^{r_{v}} & \\
T_{2}^{\prime} \leq T \leq T_{2} & R_{\mu}=\frac{T}{T_{2} p_{d} \mu^{-r_{d}}} & \\
T_{2}^{\prime} \leq T \leq 10 s & R_{\mu}=\frac{1}{p_{d} \mu^{-r_{d}}} & 1,5 \leq \mu \leq 10 \\
p_{a}=q_{a}+1 & q_{a}=3,0 \xi^{-0,3} & r_{a}=0,48 \xi^{-0,08} \\
p_{v}=q_{v}+1 & q_{v}=2,7 \xi^{-0,4} & r_{v}=0,66 \xi^{-0,04} \\
p_{d}=0.87 \xi^{0,055} & & r_{d}=1,07
\end{array}
$$




\subsection{Lai y Biggs (1980)}

Definen sismos artificiales que tienen sus espectros de respuesta elásticos compatibles para sistemas elastoplásticos. Proponen una expresión lineal en términos logarítmicos para tres regiones espectrales, en donde $\mathrm{a}$, b son valores de constantes que son función directa del rango de períodos y de los valores de ductilidades.

$R=a+b(\log T)$

\subsection{Riddell, Hidalgo y Cruz (1989)}

Los factores simplificados de reducción de resistencia fueron propuestos con base en los factores aproximados promedios de reducción de resistencia. La formulación consiste en dos tramos lineales, el primero de los cuales presenta valores variables del factor de reducción como función del valor del período esquina $\mathrm{T}^{*}$, mientras el segundo tramo, presenta un valor constante para el rango de períodos faltante.

$$
\begin{array}{ll}
0<T<T^{*} & R=1+\left(R^{*}-1\right) T / T^{*} \\
T<T^{*} & R=R^{*}
\end{array}
$$

\subsection{Nassar y Krawinkler (1992)}

Definen factores de reducción de resistencia como el inicio del desarrollo de un procedimiento de diseño que utilice la capacidad de ductilidad del miembro y la respuesta estructural inelástica como la base del diseño en el estado límite de colapso.

$$
\begin{aligned}
& R_{\mu}=[C(\mu-1)+]^{\frac{1}{C}} \\
& C=\frac{T^{a}}{1+T^{a}}+\frac{b}{T} \\
& a=0,80 \\
& b=0,29
\end{aligned}
$$

La demanda de resistencia inelástica en suelo blando es equivalente a la demanda de resistencia inelástica en perfil rocoso, multiplicado por un factor de modificación de suelo blando, el cual es función directa del período predominante del suelo y de la razón de ductilidad.

1. El espectro de diseño de resistencia elástica de suelo blando exhibe grandes amplificaciones en la vecindad del primer período del suelo. $\mathrm{El}$ ancho de estas amplificaciones aumentan conforme aumenta el valor del período predominante del suelo $\left(T_{S}\right)$.

2. La amplificación máxima de suelo blando depende débilmente del período del suelo.

La forma del espectro de diseño de resistencia inelástica es más suavizado que el espectro de diseño de resistencia elástica y los picos de los espectros inelásticos no vuelven a ocurrir en los períodos predominantes del suelo. Las zonas de amplificación inelásticas son más anchas que las elásticas.

\subsection{Miranda (1993)}

Los factores de reducción en roca se caracterizan por aumentar conforme se incrementa el valor de la razón de ductilidad, con una tasa de incremento que depende del período, y para una razón de ductilidad constante los factores de reducción de resistencia exhiben una variación importante con cambios en el período, sobre todo en región de períodos cortos. Para períodos largos, los factores de reducción de resistencia son constantes e iguales al valor de la razón de ductilidad.

Debido a la ocurrencia de la mayor amplificación para suelo blando en valores de período cercanos al período predominante del sismo, se define el período predominante del sismo $T_{g}$ como aquel período en el cual la máxima energía de entrada de un sistema lineal elástico con una razón de amortiguamiento del $5 \%$ ocurre y es 
máximo en todo el rango de períodos. El $T_{g}$ también puede corresponder al período en el cual ocurre la máxima velocidad relativa, la cual es proporcional a la energía cinética relativa, y a su vez, es similar a la energía cinética absoluta para la vecindad del período predominante (Uang \& Bertero, 1990).

Para depósito de suelo blando los factores de reducción de resistencia son mucho mayores que las razones de ductilidad para la cercanía al período $T_{g}$. Para sistemas con períodos menores o iguales a dos terceras partes del valor de $T_{g}$, el factor de reducción de resistencia es menor que el valor de la razón de ductilidad, mientras que para períodos mayores a 1,5 veces el valor de $T_{g}$, el factor de reducción de resistencia es similar al valor de la razón de ductilidad.

El factor $\Phi$ fue calibrado con los datos obtenidos de los análisis de historias en el tiempo no lineales y es definido para cada tipo de suelo

$$
\begin{aligned}
& \Phi_{\text {roca }}=1+\frac{1}{10 T-\mu T}-\frac{1}{2 T} \exp \left[-\frac{3}{2}\left(\ln T-\frac{3}{5}\right)^{2}\right] \\
& \Phi_{\text {firme }}=1+\frac{1}{12 T-\mu T}-\frac{2}{5 T} \exp \left[-2\left(\ln T-\frac{1}{5}\right)^{2}\right] \\
& \Phi_{\text {blando }}=1+\frac{T_{g}}{3 T}-\frac{3 T_{g}}{4 T} \exp \left[-3\left(\ln \left(\frac{T}{T_{g}}\right)-\frac{1}{4}\right)^{2}\right]
\end{aligned}
$$

\subsection{Riddell (1993)}

Existen dos formas de desarrollar espectros de resistencia inelásticos. La primera consiste en la reducción del espectro elástico por medio del uso de factores de reducción. La segunda consiste en la derivación directa del espectro inelástico a través de estudios estadísticos de espectros obtenidos de análisis dinámicos no lineales de estructuras. Riddell contrasta los dos métodos (indirecto y directo) llegando a la conclusión de que el procedimiento directo conduce a resultados más concordantes con las mediciones observadas. Para ello define una función en términos de un número de coeficientes $\left(a_{1}, a_{2}\right.$, $a_{3}, a_{4}, a_{5}$ ) que son determinados por análisis de regresión y que son función del suelo y de la razón de ductilidad.

$$
S_{a}(T, \mu)=\frac{a_{1}+a_{2} T^{a_{3}}}{1+a_{4} T^{a_{5}}}
$$

El efecto del suelo es importante para suelos muy blandos en períodos intermedios y bajas ductilidades, aumentando el ancho de las regiones de la amplificación de la respuesta conforme el suelo es más blando.

\subsection{Vidic, Fajfar y Fischinger (1994)}

Proponen un espectro básico que defina la demanda sísmica en el diseño por medio de cuatro componentes: espectro de resistencia (niveles de solicitación lateral), espectro de desplazamiento (comportamiento inelástico), espectro de energía de entrada (daño potencial) y espectro de energía histerética (daño acumulativo).

El factor de reducción de resistencia es, en regiones de períodos medios y largos, sólo ligeramente dependiente del período y es cercano al valor de la razón de ductilidad. Para la región de períodos cortos, el factor de reducción de resistencia depende fuertemente del período y la razón de ductilidad. Existe una moderada influencia del comportamiento histerético y del amortiguamiento en todo el rango de períodos.

Con base en estas observaciones se proponen las siguientes expresiones, en las cuales $T_{1}$ corresponde, en el espectro de factores de reducción, al período de transición de la parte dependiente del período a la parte fuertemente independiente del período; $T_{1}$ aproximadamente representa el período predominante del sismo. 


$$
\begin{array}{ll}
R_{\mu}=C_{1}(\mu-1)^{C_{R}} \frac{T}{T_{0}}+1 & T \leq T_{0} \\
R_{\mu}=C_{1}(\mu-1)^{C_{R}}+1 & T_{0} \geq T \\
T_{0}=C_{2} \mu^{C_{T}} T_{1} & \\
T=2 \pi \frac{C_{v}}{C_{a}} \frac{V}{A} &
\end{array}
$$

\subsection{Priestley (1995)}

Basado en su procedimiento de diseño sísmico sobre desplazamientos, define una relación bilineal con un período esquina que representa el período predominante del sismo.

$$
\begin{array}{ll}
R_{\mu}=1+\frac{(\mu-1)}{1.5 T_{g}} T & T \leq 1.5 T_{g} \\
R_{\mu}=\mu & T>1.5 T_{g}
\end{array}
$$

\subsection{Ordaz y Rocha (1998)}

El factor de reducción depende solamente del espectro de desplazamiento inelástico, sin necesidad de mencionar explícitamente al período de vibración. Se propone como forma básica una expresión que inicialmente se supone dependiente del factor de reducción de resistencia de la ductilidad, del espectro de velocidades relativas $\mathrm{V}(\mathrm{T})$ y del espectro de desplazamientos relativos $\mathrm{D}(\mathrm{T})$, normalizados con respecto a los valores máximos de velocidad y desplazamiento del terreno.

$$
R_{\mu}=1+\left[\frac{V(T)}{V_{\max }}\right]^{\alpha}\left[\frac{D(T)}{D_{\max }}\right]^{\beta}(\mu-1)
$$

De acuerdo con los resultados de regresión el mejor modelo está constituido por aquel que considera únicamente los desplazamientos.

$$
\begin{aligned}
& R_{\mu}=1+\left[\frac{D(T)}{D_{\text {max }}}\right]^{\beta(\mu)}(\mu-1) \quad \mathrm{D}(\mathrm{T}) \text { elástico } \\
& \beta(\mu)=a(\mu-1)^{0,173}
\end{aligned}
$$

Debido a la dificultad de contar con la información referente al desplazamiento máximo del terreno $y$ al espectro de desplazamiento relativo del sistema, se puede hacer uso de la similitud del desplazamiento máximo del terreno con el desplazamiento elástico espectral para períodos mayores a $5 \mathrm{~s}$ en suelo firme. Como alternativa se ha definido un método (Miranda, 2000) para obtener las razones de desplazamiento inelásticos, las cuales sirven para determinar la demanda inelástica de desplazamiento lateral máximo a partir de su correspondiente demanda elástica. Esto para un sistema con la misma masa y misma rigidez inicial para suelo firme.

$$
\begin{aligned}
C_{\mu} & =\frac{D_{\text {inelástico }}}{D_{\text {elástico }}} \\
C_{\mu} & =\left[1+\left[-1+\frac{1}{\mu}\right] \exp \left(-12 T \mu^{-0,8}\right)\right]^{-1}
\end{aligned}
$$

\subsection{Lee, Han y Oh (1999)}

Los modelos histeréticos (bilineal, deterioro de rigidez, deterioro de resistencia) presentan efectos que son independientes entre sí, a la hora de ser incluidos en conjunto. Para el modelo bilineal (con los parámetros de rigidez inicial, desplazamiento de fluencia y segunda pendiente) se establece una formulación que refleja la variación del factor de reducción con el valor de la segunda pendiente: a mayor valor de segunda pendiente, mayor valor de factor de reducción de resistencia.

La definición de las variables se muestran a continuación: 


$$
\begin{aligned}
& R_{\mu}=R(T, \mu) C_{1} \\
& R(T, \mu)=A_{0}\left[1-\exp \left(-B_{0} T\right)\right] \\
& A_{0}=0,99 \mu+0,15 \\
& B_{0}=23,69 \mu^{-0,83} \\
& C_{1}=1+A_{1} a+B_{1} b \\
& A_{1}=2,07 \ln \mu-0,28 \\
& B_{1}=-10,55 \ln \mu+5,21
\end{aligned}
$$

\subsection{Tiwari y Gupta (2000)}

Para suelo firme (aluvión, intermedio, roca dura) desarrollan una relación cualitativa entre el factor de reducción de resistencia con las variables de la duración del movimiento $\left(T_{s}\right)$, del período de máxima amplificación del espectro de Fourier $\left(T_{g}\right)$, del espectro de Fourier (FS $(T)$ ) y del valor de la aceleración máxima del terreno (PGA).

$$
R_{\mu}=10^{\alpha(T) T_{g}} T_{s}^{\beta(T)} \frac{F S(T)}{P G A}
$$

Los valores de factor de reducción de resistencia bajos se asocian con sismos de larga duración, excepto para períodos medios.

\subsection{Manfredi (2001)}

El factor de reducción de resistencia es numéricamente igual a la razón de la fuerza elástica con la fuerza de fluencia para un nivel dado de razón de ductilidad. Se define un parámetro $x=T / T_{1}$ (para $T<T_{1}$ ) donde $T$ es el período elástico y $T_{1}$ es el período inicial del rango de períodos medios, igual a $1 \mathrm{~Hz}$.

$$
R_{\mu}=m \frac{S_{a}(T)}{F_{y}}
$$

\section{SELECCIÓN DEL CRITERIO DE OBTENCION DE FACTORES DE REDUCCIÓN}

Todo criterio para la obtención de los factores de reducción de resistencia dependiente de la ductilidad debe cumplir las siguientes condiciones (Miranda \& Bertero, 1994):

1. Para el caso elástico (razón de ductilidad igual a uno) el factor de reducción de resistencia debe ser igual a uno, ya que no existe incursión inelástica, y por lo tanto, no debe existir reducción alguna de la demanda de resistencia.

2. La demanda de resistencia inelástica es la misma que la demanda de resistencia elástica para aquellos sistemas con valores de períodos cercanos a cero, o sea sistemas rígidos. Por lo tanto, el factor de reducción de resistencia debe ser igual a uno.

3. El desplazamiento máximo relativo tiende al valor del máximo desplazamiento del terreno para sistemas con valores de períodos muy grandes, o sea, sistemas flexibles. La demanda de resistencia inelástica es igual a la demanda de resistencia elástica dividida por la razón de ductilidad, lo que implica que el factor de reducción de resistencia debe tender al valor de la razón de ductilidad.

4. Para períodos cercanos al período predominante del sismo, en sistemas cimentados sobre perfiles de suelo blando, los factores de reducción de resistencia son mayores que los valores de razón de ductilidad, esto debido al efecto de amplificación de la señal sísmica.

5. El factor de reducción de resistencia debe ser dependiente del período.

El criterio de Newmark y Hall no presenta una forma clara de representar el efecto del suelo, salvo las relaciones V/A y $\mathrm{AD} / \mathrm{V}^{2}$ las cuales son consideraciones que no cumplen bien para 
perfiles de suelo blando. Además para un gran rango de períodos el factor de reducción de resistencia es independiente del valor del período de vibración.

El criterio de Lai y Biggs para una ductilidad alta de 4 no tiende, en la zona de períodos largos, al valor de la razón de ductilidad. Y no presenta evidencia del efecto del suelo.

El criterio de Riddell y Newmark, el cual es utilizado íntegramente por el CSCR-2002 para la determinación de los espectros inelásticos de diseño, presenta valores del factor de reducción de resistencia mayores que el valor de la razón de ductilidad para períodos altos. Aunque tiene una leve mejoría en cuanto a la disminución de las zonas independientes del valor del período, sigue sin evidenciar el efecto del suelo sobre la respuesta, salvo por el uso de las relaciones espectrales mencionadas en el caso del criterio de Newmark y Hall.

El criterio de Riddell, Hidalgo y Cruz, a pesar de seguir con la línea de definición de los criterios de Newmark, logra encontrar el valor de la razón de ductilidad para períodos altos.

ElcriteriodeNassary Krawinklerparaductilidades altas, sugiere algún tipo de amplificación por efecto de la condición del suelo, pero dicha presencia es leve y se distorsiona en valores mayores a la razón de ductilidad para períodos altos.

Miranda presenta consideraciones de factores de reducción de resistencia dependientes de las condiciones del suelo de cimentación . En los tres tipos de suelo y con mayor énfasis en el suelo blando es evidente la presencia de la amplificación de la respuesta.

Riddell no presenta valores que puedan corresponder a un comportamiento lógico, e inclusive en el suelo $S_{3}$ presenta una incongruencia numérica (factores de reducción de resistencia con valores negativos) para períodos superiores a $2 \mathrm{~s}$.
Vidic y Fajfar continúan con la tendencia de definir relaciones bilineales, las cuales, en este caso, no cumplen con los límites para períodos altos ni presentan un efecto claro de la condición del suelo.

Priestley defiende la línea de definición de Vidic y Fajfar, con los mismos resultados, aunque en este caso sí cumple con el valor de la razón de ductilidad para períodos altos.

Lee, Han y Oh presentan un comportamiento similar al de Nassar y Krawinkler, pero con el gran aporte de insistir en el efecto que tiene el modelo histerético seleccionado. Presenta para el perfil rocoso un comportamiento similar al de Miranda.

Manfredi y Cosenza no dan mayor aporte, ya que sus relaciones numéricas no se cumplen para períodos cortos.

Con la excepción de no haber tomado en cuenta el criterio de Ordaz y Rocha, por la dificultad de contar con un espectro de desplazamientos elásticos que represente una tendencia entre los registros que conforman la base de datos del estudio, es el criterio de Miranda el que presenta el mejor comportamiento para ser considerado como el modelo a seguir para la obtención de los factores de reducción de resistencia, esto con base en la capacidad del criterio de representar los factores de reducción para los tres distintos tipos de suelo y por cumplir las condiciones establecidas con anterioridad

\section{COMPARACIÓNENTREELCRITERIO DE MIRANDA Y EL CSCR-2002}

El CSCR-2002 utiliza los criterios de RiddellNewmark (1979) para definir los factores de reducción de resistencia para amortiguamientos del $5 \%$, utilizando sismos registrados en roca y aluvión. Los factores dependen del valor de la ductilidad de desplazamiento, la región espectral y la razón de amortiguamiento. La consideración del tipo de suelo se define en las razones V/A y $\mathrm{AD} / \mathrm{V}^{2}$, Figura 2. 
Para sistemas muy flexibles (períodos altos) el desplazamiento máximo relativo tiende hacia el desplazamiento máximo del terreno. Además, la demanda inelástica de resistencia es igual a la demanda elástica de resistencia dividida por la razón de ductilidad de desplazamiento y el factor de reducción de resistencia debe tender al valor de la ductilidad. Dicha condición no es satisfecha por el criterio de Riddell-Newmark, En caso contrario a Miranda. Por esta razón y por considerar el efecto de suelos blandos, se considera que Miranda presenta criterios más adecuados que los utilizados por el CSCR2002.

Para el caso de los factores de reducción de resistencia en perfil rocoso, mostrado en la Figura 2a, existe una discrepancia importante entre Riddell-Newmark y Miranda (mayor valor para Miranda) para el rango de períodos $0,75 \mathrm{~s} \mathrm{a}$ $3 \mathrm{~s}$. La máxima diferencia ocurre en período $1,5 \mathrm{~s}$ aumentando la magnitud de la diferencia máxima conforme aumenta la ductilidad. Para períodos mayores a 3,2 s el criterio de Miranda queda por debajo de Riddell-Newmark, específicamente por la tendencia de Miranda de aproximarse al valor de la ductilidad correspondiente.

Para el caso de factores de reducción de resistencia en perfil de suelo firme, indicado en la Figura 2b, existe la discrepancia de valores para períodos inferiores a 2,75 s para ductilidades 3,4 y 6 . Para las ductilidades pequeñas $(1,5$ y 2) el límite de período se traslada a valores de períodos pequeños, disminuyendo también el valor máximo de la discrepancia. La mayor discrepancia a favor de Miranda para todas las ductilidades ocurre para el período de $1 \mathrm{~s}$.

Para el caso de los factores de reducción de resistencia en perfil de suelo blando, indicado en la Figura 2c, se ha definido como valor de período fundamental de sismo al período promedio para los registros que corresponden a perfiles de suelo $\mathrm{S}_{3}$ y $\mathrm{S}_{4}$, que corresponde a 1 s. Se presenta un comportamiento similar a los perfiles de roca y firme, en cuanto a la presencia de las zonas de discrepancia entre los criterios.
La mayor diferencia a favor de Miranda ocurre en período de $1 \mathrm{~s}$, manteniéndose similar el rango de períodos en el cual ocurren las diferencias.

Para los tres tipos de suelos, la magnitud de la discrepancia se incrementa conforme el suelo va de roca hasta suelo blando. En las Figuras 3a, $3 \mathrm{~b}, 3 \mathrm{c}$ y $3 \mathrm{~d}$ se presenta el comportamiento para la zona sísmica Z3 definida en el CSCR-2002, correspondiente a un valor de aceleración pico efectiva de $0,30 \mathrm{~g}$.

En los tres casos anteriores, para zonas de períodos cortos e intermedios el CSCR-2002 presenta valores de reducción de resistencia menores a los de Miranda, lo que significa la presencia en los espectros del CSCR-2002 de valores de coeficiente sísmico mayores a los que, según el criterio de Miranda, se van a presentar a la hora de construir los espectros inelásticos.

Para los espectros en suelo rocoso indicados en la Figura 3a, en todas las ductilidades presentan dos zonas en las cuales ocurren los valores máximos de diferencias a favor de Miranda (valor con Miranda es mayor al del CSCR-2002) para períodos desde $0,15 \mathrm{~s}$ hasta $0,4 \mathrm{~s}(25 \%$ aproximadamente para cada caso), y una sola zona en donde la diferencia está a favor del CSCR-2002 con su valor máximo en período de 1,65 s ( $5 \%$ hasta $25 \%$ conforme aumenta la ductilidad).

Para los espectros en suelo firme indicados en la Figura 3b, se presentan dos zonas en las cuales Miranda tiene el valor mayor, precisamente al inicio y al final del rango total de períodos. Conforme aumenta la ductilidad, estos intervalos de períodos se van acortando para pasar de 0,3 $\mathrm{s}$ en ductilidad 1,5 a $0,15 \mathrm{~s}$ en ductilidad 6 , y para pasar de $1,9 \mathrm{~s}$ en ductilidad 1,5 a $3,3 \mathrm{~s}$ en ductilidad 3; para ductilidades 4 y 6 existe un alargamiento del intervalo hasta llegar al valor de $2,8 \mathrm{~s}$.

Para los espectros en suelo blando indicados en la Figura 3c, se definen dos zonas en las cuales Miranda presenta el valor mayor (al inicio y 
final del rango total de períodos) con los picos presentándose en períodos 0,14 s y 4 s (40\% y 5 $\%)$, conforme aumenta la ductilidad la magnitud del pico de 0,14 s va incrementando su valor. Los rangos de períodos en los cuales Miranda tiene el valor mayor se van haciendo más estrechos conforme aumenta la ductilidad, pasando de 0,6 s y 1,75 s en ductilidad 1,5 a 0,25 s y 4 s en ductilidad 6 .

Similar comportamiento al del suelo blando, ocurre en los espectros en suelo muy blando, indicados en la Figura 3d.

\section{CONCLUSIONES}

1. El factor de reducción por resistencia (utilizado por el CSCR-2002) no cumple con tres aspectos importantes: a) función dependiente del período, b) presencia de amplificación de la respuesta debida a la condición de sitio de cimentación para condición de sueloblando,c) comportamiento asintótico del factor de reducción al valor de la razón de ductilidad para períodos altos (estructuras flexibles).

2. El criterio definido por Miranda es el único de los criterios estudiados que cumple con todos los requisitos esperados, para una formulación matemática que pueda conducir a la obtención de factores de reducción de resistencia.

3. La utilización de la razón espectral V/A no representa una forma adecuada de considerar el efecto del sitio de cimentación en la respuesta, debido a la inexistencia de una variación en el comportamiento en la zona cercana al período predominante del sismo, zona en la cual se producen amplificaciones que exceden el comportamiento previsto por el criterio de Riddell y Newmark.

4. La condición de suelo blando es la que presenta la mayor discrepancia en la comparación de los valores de los factores de reducción de resistencia de Miranda con el utilizado por el CSCR-2002.
5. La utilización del criterio de Miranda produce mayores valores del factor de reducción de resistencia, lo que implica valores del FED menores a los definidos en los espectros de diseño del CSCR-2002. Esto conduce a considerar niveles de solicitaciones laterales sísmicas inferiores a las definidas tomando, en cuenta el efecto del suelo de cimentación.

6. La mayor amplificación de la respuesta obtenida con el uso del criterio de Miranda se concentra, para los tres tipos de suelos de cimentación, en el intervalo de períodos de $1,0 \mathrm{~s}$ a $1,5 \mathrm{~s}$, zona que coincide con el valor promedio del período predominante del sismo, para el caso de perfiles de suelo blando o muy blando.

7. Al incorporar el criterio de Miranda a las formas espectrales del CSCR-2002, ocurre un incremento de los valores del FED para la zona de períodos muy cortos y disminuyen los valores en el rango de períodos de la amplificación de la respuesta.

8. El criterio de Miranda no debe ser considerado el único acertado o el que tenga la mejor definición, pero la discrepancia de valores con el CSCR-2002 plantea la necesidad de realizar un trabajo orientado hacia la comparación entre ambos criterios, para determinar si es necesario construir un criterio híbrido que responda a eliminar dichas diferencias.

\section{REFERENCIAS BIBLIOGRÁFICAS}

Chopra, A. (1981). Dynamics of structures: A Primer. Earthquake Engineering. California, United States of America.: Research Institute.

Chopra, A. (1995). Dynamics of structures: theory and applications to earthquake engineering. New Jersey, N.Y.: Prentice Hall.

Colegio Federado de Ingenieros y Arquitectos, (2002). Gutiérrez, J. (editor). Código 
Sísmico de Costa Rica. Versión 2002. Cartago: Editorial Tecnológica de Costa Rica.

Cruz, M., Infante, E. \& Schmidt. V. (1998). Evaluación del potencial de daño de sismos recientes en Costa Rica, Memorias presentadas delX SeminarioLatinoamericano de Ingeniería Sísmica (pp. 163-174). San José, Costa Rica.

Fajfar, P. (1992). Equivalent ductility factors, taking into account low-cycle fatigue. Earthquake Engineering and Structural Dynamics, 21, 837-848.

Fajfar, P. (1995). Elastic and inelastic design spectra. En: 10 ${ }^{\text {th }}$ European Conference on Earthquake Engineering, (pp. 1169-1178). Balkema, Rotterdam.

Gupta, A. (1992). Response spectrum method in seismic analysis and design of structures, CRC Press. Florida, Estados Unidos de América.

Jiménez, O. (1996). Espectros inelásticos del terremoto de Limón de 1991. Trabajo de Graduación para Licenciatura en Ingeniería Civil, Escuela de Ingeniería, Universidad de Costa Rica, San José, Costa Rica.

Kawashima, K., Hoshikuma, J. \& Nagaya, K. (1998). Residual displacement response spectra. Journal of Structural Engineering, 124(5), 523-530.

Krawinkler, H. \& Rahnama, M. (1995). Effects of soft soils on design spectra. En: $10^{\text {th }}$ European Conference on Earthquake Engineering, (pp. 5841-5846). Balkema, Rotterdam.

Lai, S. \& Biggs, J. (1980). Inelastic response spectra for aseismic building design. Journal of Structural Division ASCE, 106(ST6), 1295-1310.

Lam, N., Wilson, J. \& Hutchinson, G. (1998). The ductility reduction factor in the seismic design of buildings. Earthquake Engineering and Structural Dynamics, 27, 749-769.

Lee, L., Han. S. \& Oh. Y. (1999). Determination of ductility factor considering different hysterestic models. Earthquake Engineering and Structural Dynamics, 28, 957-977.

Mahin, S. \& Bertero, V. (1981). An evaluation of inelastic seismic design spectra. Journal of the Structural Division, 107(9), 1777-1795.

Mahin, S. \& Lin, L. (1983). Construction of inelastic response spectra for single-degreeof-freedom systems, USA: University of California, Earthquake Engineering Research Center.

Miranda, E. (1993). Site-dependent strengthreduction factors. Journal of Structural Engineering, 119 (12), 3503-3519.

Miranda, E. (1995). Nonlinear response spectra for earthquake resistant design. En: $10^{\text {th }}$ European Conference on Earthquake Engineering. (pp. 5835-5840). Balkema, Rotterdam.

Miranda, E. (2000). Inelastic displacement ratios for structures on firm sites. Journal of Structural Engineering, 126 (10), 11501159.

Miranda, E. \& Bertero, V. (1994). Evaluation of strength reduction factors for earthquakeresistant design. Earthquake Spectra, 10 (2), 357-379.

Miranda, E. \& Ruíz-García, J. (2002). Evaluation of approximate methods to estimate maximum inelastic displacement demands. Earthquake Engineering and Structural Dynamics, 31, 539-560.

Directorate of Licensing U.S. Atomic Energy Commission (1973). A study of vertical and horizontal earthquake spectra (Report No. WASH-1255). Mohraz, B., Hall, W. \& Newmark, N.

Nassar, A., Osteraas, J. \& Krawinkler, H. (1995). Seismic design based on strength and ductility demands. En: $10^{\text {th }}$ European Conference on Earthquake Engineering, (pp. 5861-5866). Balkema, Rotterdam.

Newmark, N. \& Hall, W. (1973). Seismic design criteria for nuclear reactor facilities (pp. 209-236) (Report 46). Building Practices for Disaster Mitigation, National Bureau of Standards, U.S. Department of Commerce. 
Newmark, N. \& Hall, W. (1982). Earthquake Spectra and Design. California, United States of America: Earthquake Engineering Research Institute.

Ordaz, M. \& Perez-Rocha, L. (1998). Estimation of strength-reduction factors for elastoplastic systems: a new approach. Earthquake Engineering and Structural Dynamics, 27, 889-901.

Ramírez, R., Santana, G., Chacón, O. (1995). Mapa de amplificación sísmica del Valle Central. San José, Costa Rica.

Riddell, R. \& Newmark, N. (1979). Statistical analysis of the response of nonlinear systems subjected to earthquakes Structural Research Series No. 468. Urbana, USA: University of Illinois, Department of Civil Engineering.

Riddell, R. (1989). Response modification factors for earthquake resistant design of short period buildings. Earthquake Spectra, 5(3), 571-590.

Riddell, R. (1995). Inelastic design spectra accounting for soil conditions. Earthquake Engineering and Structural Dynamics, 24, 1491-1510.

Santana, G. \& Terán-Gilmore, A. (1994). Damage potential of earthquake ground motions recorded in southern Central America. Revista de Ingeniería de la Universidad de Costa Rica, 4(2), 75-88.

Sawada, T., Hirao, K. \& Yamamoto, H. (1995). Relation between maximum amplitude ratio $\left(\mathrm{A} / \mathrm{V}, \mathrm{AD} / \mathrm{V}^{2}\right)$ and spectral parameters of earthquake ground motion. En: $10^{\text {th }}$ European Conference on Earthquake Engineering, (pp. 617-622). Balkema, Rotterdam.

Seed, H. \& Idriss, I. (1971). Influence of soil conditions on building damage potential during earthquakes. Journal of Structural Division, 97(2), 639-663.

Tiwari, A. \& Gupta, V. (2000). Scaling of ductility and damage-based strength reduction factors for horizontal motions. Earthquake Engineering and Structural Dynamics, 29, 969-987.

Uang, C. (1991). Establishing R (or Rw) and Cd Factors for building seismic provisions. Journal of Structural Engineering, 117(1), 19-28.

Uang, C. (1995). Seismic force reduction and displacement amplification factors. En: $10^{\text {th }}$ European Conference on Earthquake Engineering, (pp. 5875-5880). Balkema, Rotterdam.

Veletsos, A. \& Newmark, N. (1960). Effects of inelastic behavior on the response of simple systems to earthquake ground motions. En: 2nd World Conference on Earthquake Engineering, (pp. 895-912). Japan.

Vidic, T., Fajfar, P. \& Fischinger, M. (1994). Consistent inelastic design spectra: strength and displacement. Earthquake Engineering and Structural Dynamics, 23, 507-521.

Whittaker, A., Hart, G. \& Rojahn, C. (1999). Seismic response modification factors. Journal of Structural Engineering, 125(4), 438-444.

\section{SOBRE LOS AUTORES}

\section{Sc. Rubén Leandro Ulloa.}

Magister Scientaiae en Ingeniería Civil con énfasis en Estructuras, de la Universidad de Costa Rica, investigador independiente Teléfonos: 392-7676 y 237-1570

Facsímil: 261-2382

Correo electrónico: rlulloa@cfia.or.cr; ruben.leandro@amanco.com

\section{Ph. D. Guillermo Santana Barboza.}

Ingeniero civil, profesorcatedrático investigador del Sistema de Estudios de Posgrado de la Escuela de Ingeniería Civil, de la Universidad de Costa Rica..

Teléfono: 207-4012

Facsímil: 207-4440

Correo electrónico: santana@lanamme.ucr.ac.cr 

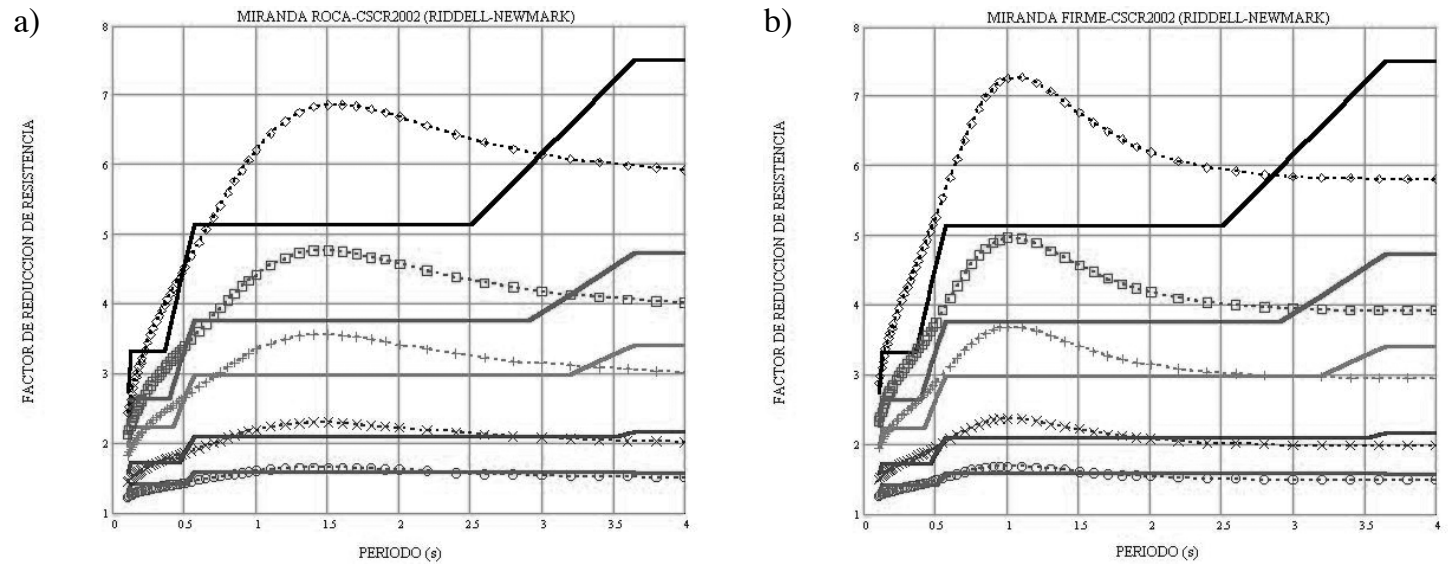

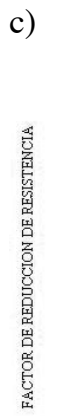
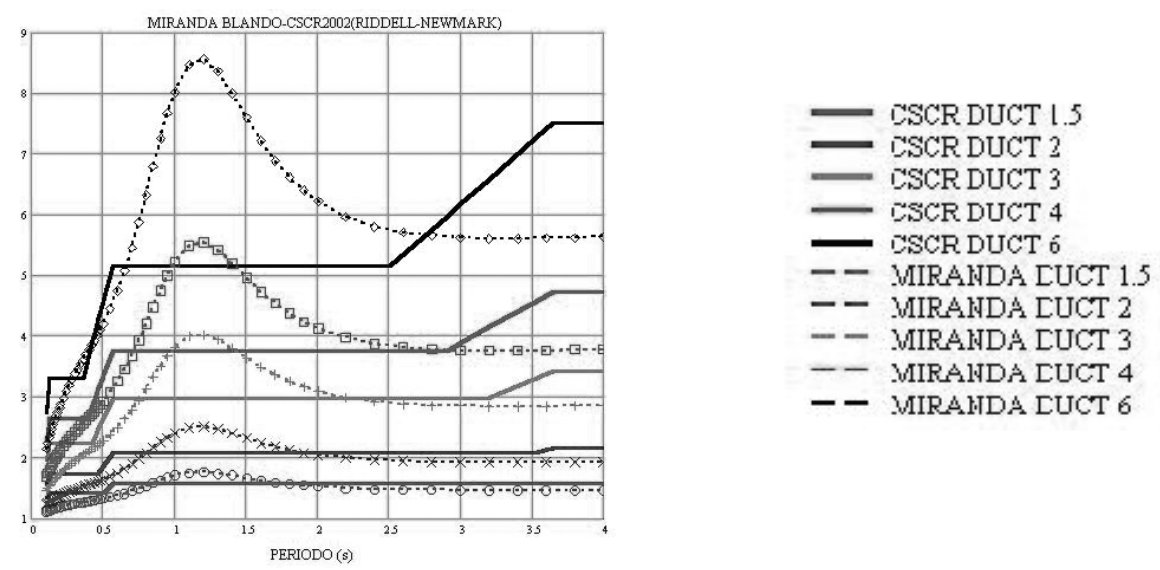

Figura 2: Comparación de valores de factores de reducción de resistencia: a) Miranda para suelo rocoso versus CSCR-2002, b) Miranada para suelo firme versus CSCR-2002, c) Miranda para suelo blando versus CSCR-2002)

Ver imágenes a color en la página 165. 
a)

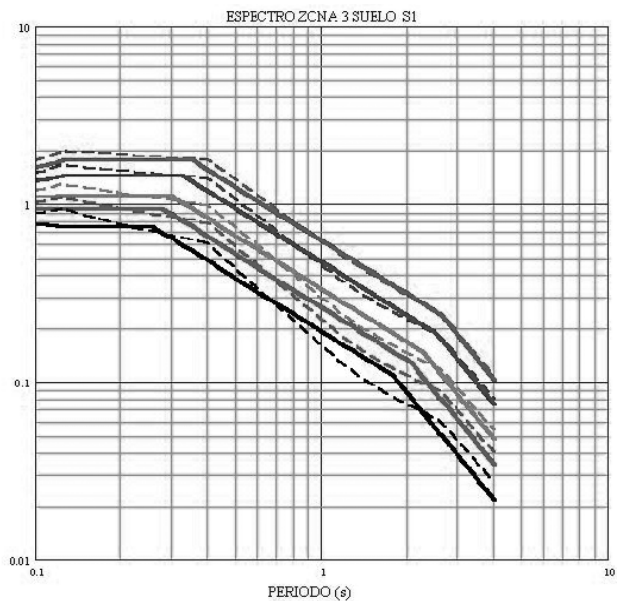

c)

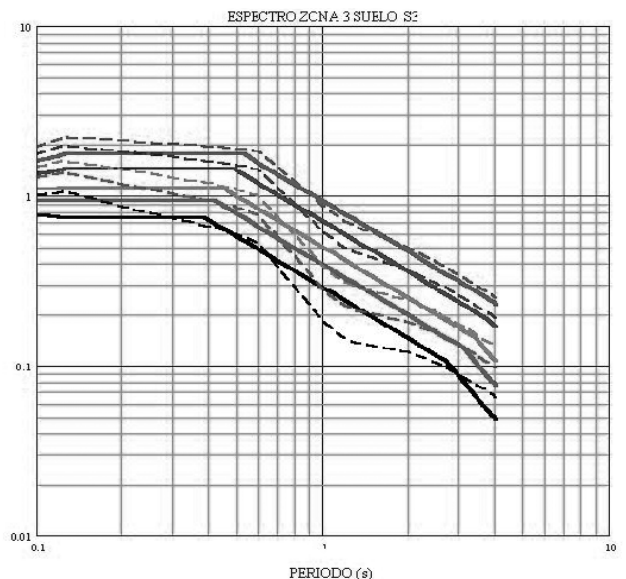

b)

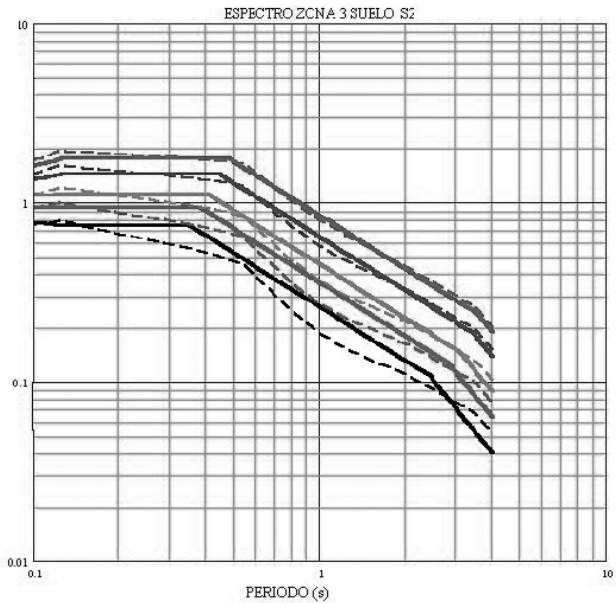

d)
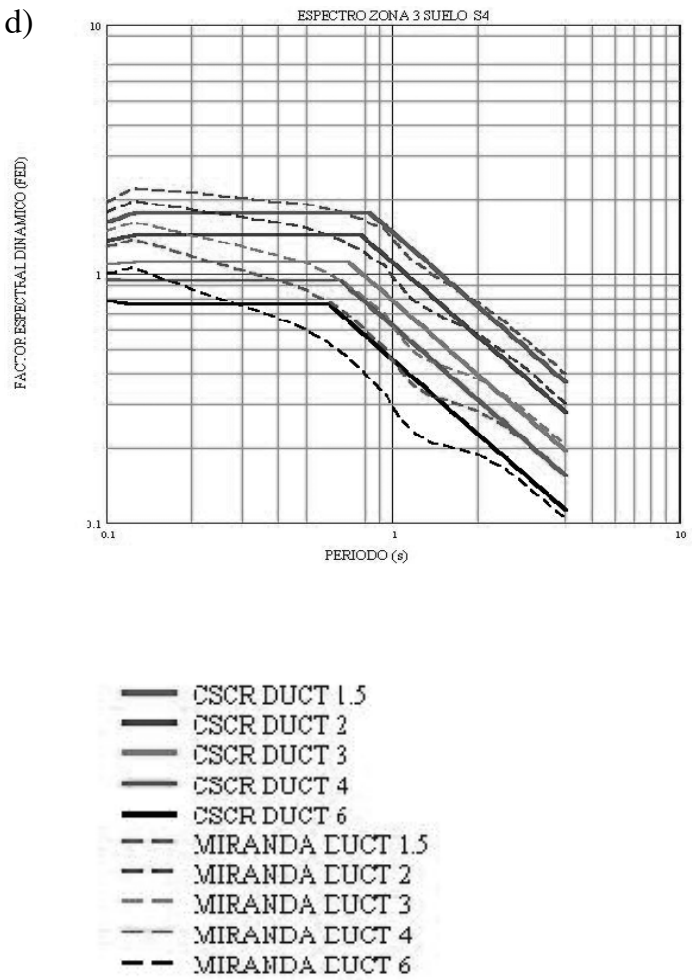

Figura 3: Comparación del espectro de diseño Zona sísmica y suelo cimentación (Miranda versus CSCR-2002): a) Z3 S1, b) Z3 S2, c) Z3 S3, d) Z3 S4.

Ver imágenes a color en la página 166. 